TP Periodica Polytechnica

Electrical Engineering and Computer Science

58(3), pp. 109-120, 2014

DOI:10.3311/PPee.7353

Creative Commons Attribution (i)

RESEARCH ARTICLE

\section{Resistance Estimation in Solenoid Actuators by Considering Different Resistances in the PWM Paths}

\author{
Ivor Dülk / Tamás Kovácsházy
}

RECEIVED 13 February 2014; ACCEPTEd AFTER REVISION 29 MAY 2014

\begin{abstract}
The estimation of the resistance of solenoid actuators is important to reduce bias in model based control and estimation as the resistance of the coil can change significantly with temperature. Under PWM operating conditions, the overall resistance that the current encounters during the "on" and "off" periods of the PWM cycles can be different because of non idealities, e.g., the resistance of wires and junctions of the switching transistors. In this paper, methods are presented for estimating the resistance of solenoid actuators by considering the difference between the overall resistances in the energizing paths. First, the steady-state PWM waveform of the coil's current is considered. Then, methods are presented for the transient situation; thus, the resistance can be estimated in case the control signal is changing. Due to the fact that solenoid actuators are usually implemented in embedded applications which have strict resource limitations, the low complexity and computational load were key requirements. Computer simulations and experimental results are also presented.
\end{abstract}

\section{Keywords \\ resistance $\cdot$ estimation $\cdot P W M \cdot$ solenoid}

\section{Introduction}

Knowledge and tracking of the model parameters of electromechanical devices are important for achieving high effectiveness at sensorless control applications, because the parameters of the model may change during normal operation. From the viewpoint of possible sources of parameter sensitivity, temperature has a major role as it can cause the resistance of the coil of electromagnetic devices, e.g. solenoid actuators, to increase by $40 \%$ for a $100^{\circ} \mathrm{C}$ temperature rise. Therefore, the measurement or estimation of the electrical resistance of the coil is important for reducing bias in control and sensorless schemes which rely on a model of the actuator. Because of the fact that cost effectiveness is a major principle in engineering practice, especially in embedded applications, model based estimation methods are preferred than direct measurements with dedicated external sensors.

For rotary induction machines the literature on estimating the resistance of the windings is comprehensive; some major contributions include [1-3]. However, for solenoid actuators the methods that are available for rotary motors are not applicable; because solenoid actuators have a single phase structure, they lack cyclic signals and have unique electrical drive conditions [4]. Solenoid actuators (Fig. 1) are electromechanical converters with a linear limited travel and they are most commonly used for flow controlling purposes and as contactors. A review of literature has shown that the sensorless methods, which are available for solenoid actuators, do not satisfactorily consider the variations in the coil's resistance caused by thermal effects [4-9], [13]. Therefore, efficient methods for estimating the resistance of solenoid actuators are of great importance for improving robustness and effectiveness. Furthermore, an estimate of the resistance of the winding can also provide information about the thermal state of the actuator.

In [11] the resistance is estimated from a lumped, dynamic thermal model that is continuously evaluated accordingly to the internal and external thermal boundary conditions. A possible drawback of [11] is that the estimate of the resistance is susceptible to the parameters of the thermal model, to the 

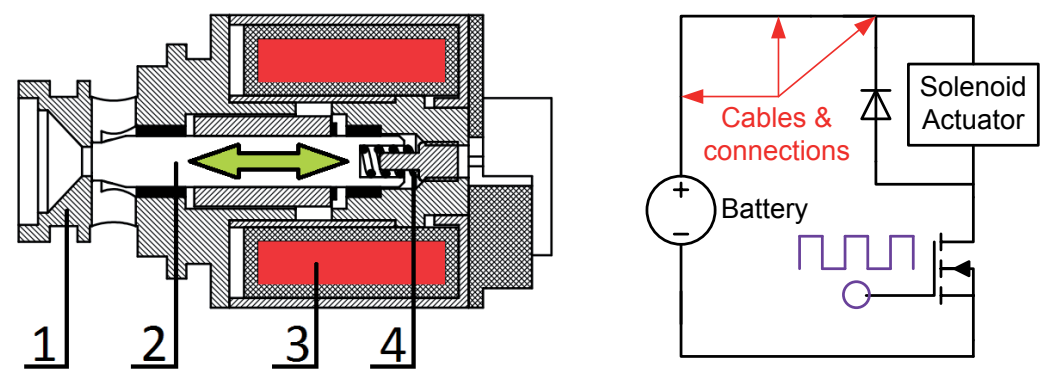

Fig. 1. Schematic of a solenoid actuator and an exemplary PWM drive configuration. 1-orifice, 2-spool, 3-coil, 4-return spring.

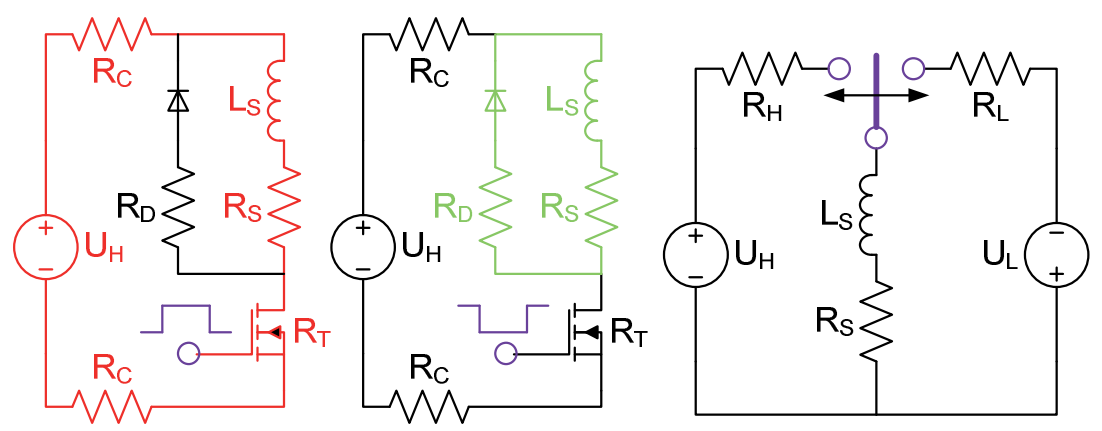

Fig. 2. Model of a non-ideal PWM drive configuration (subsidiary resistances) and generalization (right).

external thermal boundary conditions e.g. ambient temperature which require extra sensors or additional models to be measured, and to the initial conditions. A detailed thermal model is also presented in [10] but it has the same disadvantages as [11]; and may become computationally exhaustive, which is not advantageous in embedded applications. An alternative method is presented in [12] for PWM driven solenoids that directly estimates the resistance from the electrical signals; thus, the aforementioned modeling problems are avoided although the electrical model considers a simple LR model. Since solenoid actuators are most commonly used in embedded applications, the complexity and the computational needs of the methods for the estimation of the resistance have to be as low as possible.

In engineering practice, a common way for driving solenoid actuators is by means of PWM (pulse width modulation) in a single switch battery powered configuration, as it is illustrated in Fig. 1 (e.g. in the automatic transmission unit of a car). According to Fig. 1, the solenoid's coil current flows through different sections of the circuit (energizing paths) during the "on" and "off" periods of each PWM cycle. Considering real applications, the voltage source, the connecting cables, the connections, the switching transistor and the PCB (printed circuit board) all have some resistance, which add up to each other and to the resistance of the coil. Therefore, the current of the actuator encounters different overall resistances in the energizing paths and; by only measuring electrical signals, it is the separate overall resistances that can be estimated. In engineering applications, the difference between the resistances of the energizing paths can be comparable to the resistance of the winding, e.g. $0.5 \mathrm{Ohms}$ to $4 \mathrm{Ohms}$. If this difference is neglected, then the estimate of the coil's resistance may be subject to significant error; and thus the other estimates which depend on the estimate of the resistance also become biased, e.g. the temperature of the winding.

In this paper, methods are developed for estimating the resistance of the coil of solenoid actuators from both steady and transient PWM signals; with consideration to the resistance difference between the PWM "on" and "off" paths. Thus, a better estimate of the coil's resistance can be provided compared to computing a single "equivalent" resistance. The special requirements of embedded systems, e.g. limited memory and CPU capacity, are also considered at developing the methods for the estimation of the resistance.

\section{Statement of the problem}

A solenoid actuator is driven by PWM in a low-side single switch configuration as it is illustrated in Fig. 1. By measuring only the main electrical signals, i.e., supply voltage and coil current; its resistance is to be estimated for compensating the changes in the resistance which are caused by e.g. thermal effects. In practice, the voltage supply, the switching transistor, the connecting wires and the electrical connections have resistances which add to each other and to the resistance of the coil. Therefore, the separate energizing paths, between which the coil current commutates in each PWM cycle, have different total resistances. If the switch is turned on (PWM "on"), the coil is energized and its current flows from the supply through the switching transistor and through some connecting wires, as it is illustrated in the left-hand side of Fig. 2. If the switch is turned off (PWM “off”) the coil's current commutates through 
the freewheeling diode and flows through a different path that is illustrated in the middle side of Fig. 2. In Fig. 2 the terms $R_{C}$ and $R_{D}$ refer to the resistances of cables and connections, $R_{T}$ refers to the junction resistance of the switching transistor, $R_{S}$ and $L_{S}$ refer to the resistance and inductance of the solenoid respectively and $U_{H}$ refers to the supply voltage. The drive PWM has a time period $T$ and duty ratio $d$, and the sampling frequqency is considerably higher than the switching one.

The aforementioned problem can be generalized accordingly to the right-hand side of Fig. 2, where $R_{H}$ and $R_{L}$ denote the sum of the corresponding subsidiary resistances and $R_{S}$ denotes the coil's resistance. In each energizing path, the solenoid is subject to the $U_{H}$ (PWM on) and $U_{L}$ (PWM off) supply voltages, respectively. Considering Fig. 2, the $U_{L}$ is represented as the forward voltage drop on the freewheeling diode.

The resistances which can be estimated are the overall resistances in the energizing paths which consist not only of the coil's resistance; therefore, they are always higher than (or equal to) the coil's resistance. If the resistance difference between the overall resistances is not considered but a single "equivalent" resistance is estimated, then the estimate of the resistance will be between the values of the overall resistances of the energizing paths. Furthermore, it will also depend on the duty ratio thus a bias is induced which depends on the duty ratio. However, if the difference in the overall resistances is considered, then a better estimate of the coil's resistance can be provided which will correspond to the lower one of the resistances of the energizing paths, i.e., $R_{H}+R_{S}$ or $R_{L}+R_{S}$ in Fig. 2. Further on, the overall resistances of the energizing paths are denoted as in (5).

In the following sections, we differentiate between two situations. In Section 3, the overall resistances of the energizing paths are estimated from the PWM steady-state signals. The electrical model of the solenoid is assumed to be a series inductance-resistance circuit with an inductance that depends on the current; however, eddy current effects are negligible. Because the steady-state waveform is considered the back EMF (electromotive force) has no significance.

In Section 4, the overall resistances of the energizing paths are estimated from the transient electrical signals, before steady-state is reached. Thus, if the system undergoes a long transient period, i.e., the control signal changes, resistance data can be still provided. Compared to the previous modeling assumptions, the inductance is considered as a linear, constant inductance and eddy currents are negligible. From a modeling viewpoint, the effect of EMF can be represented as voltage source to the electrical system. For the methods in section 4, the EMF is considered to be negligible or as a constant voltage input. In case of solenoid actuators (or contactors), the stroke, i.e., the total change in the working air gap can be relatively small (less than one millimeter [13]) thus the change in the magnetic reluctance is small [4]; enabling the EMF to be omitted. For actuators which have larger stroke, this assumption can pose some limitation. However, it is possible to compensate the supply voltage input with the EMF part by using a previously defined EMF model and measuring the velocity of the moving part. Considering the fact that mechanical time constants are usually much larger than electrical time constants, it can be reasonable to assume that the changes in the velocity of the spool and in the position dependent magnetic reluctance are insignificant during a single estimation; thus, the EMF voltage can be considered to be constant. Then, the methods in section 4 apply.

In both situations (sections 3 and 4), the resistance depends only on temperature; and the thermal time constant of the system is considered to be larger than the electrical time constants; therefore, changes in the resistance are negligible during the period of a single estimation.

\section{The Steady-State Based Method}

In the steady-state of the PWM the coil current fluctuates between a high and low level which are unchanged during the PWM cycles. Additionally, the overall flux change in a PWM cycle must be zero; otherwise it would lead to accumulation in magnetic energy thus increase in current (continuity of flux). An exemplary steady-state PWM current waveform, with a hysteretic inductance function, is illustrated in Fig. 3.

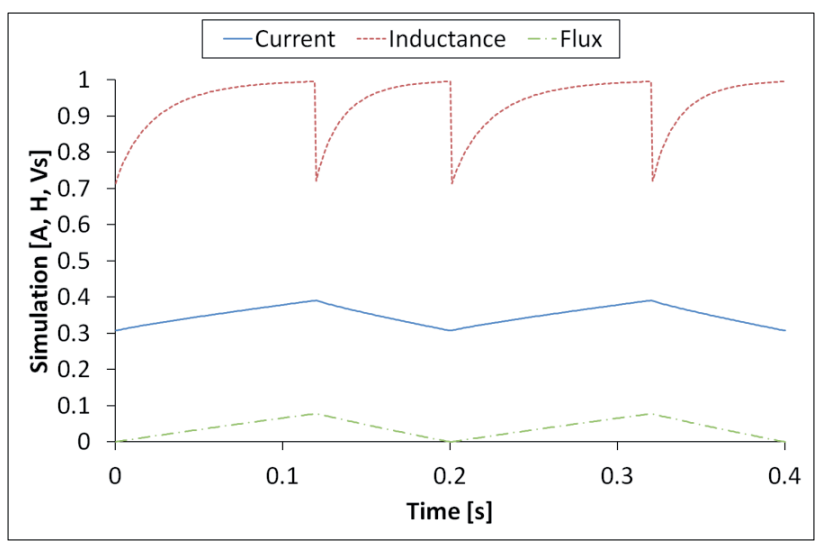

Fig. 3. Exemplary steady state PWM current waveform with hysteresis in the inductance

Using the voltage induction law for the PWM "on" and "off" periods and exploiting flux balance; the governing equations become (1)-(5). The symbol $d$ refers to the duty ratio, and $T$ refers to the time period of a PWM cycle, and $\Psi$ refers to the magnetic flux.

$$
\begin{gathered}
\Delta \Psi_{H}=\int_{i_{L}}^{i_{H}} L_{H}(i) \cdot d i=\int_{0}^{d \cdot T}\left(U_{H}(t)-R_{A} \cdot i(t)\right) \cdot d t \\
\Delta \Psi_{L}=\int_{i_{H}}^{i_{L}} L_{L}(i) \cdot d i=\int_{d \cdot T}^{T}\left(U_{L}(t)-R_{B} \cdot i(t)\right) \cdot d t \\
\Delta \Psi_{H}+\Delta \Psi_{L}=0
\end{gathered}
$$




$$
R_{A} \cdot \int_{0}^{d \cdot T} i(t) \cdot d t+R_{B} \cdot \int_{d \cdot T}^{T} i(t) \cdot d t=\int_{0}^{d \cdot T} U_{H}(t) \cdot d t+\int_{d \cdot T}^{T} U_{L}(t) \cdot d t
$$

$$
R_{A}=R_{H}+R_{S} \quad R_{B}=R_{L}+R_{S}
$$

According to (4), the inductance function has no effect on estimating the resistances from the steady-state waveform because only the average supply voltage and average current values are that matter. Because (4) expresses a linear combination of the resistances at a given supply voltage and duty ratio setting, multiple steady-state current waveforms are necessary, which are captured at different supply voltages or duty ratios. From the successive evaluation of (4), a system of linear equations can be formulated (6), the solution of which yields the overall resistances in the energizing paths.

$$
\begin{aligned}
& {\left[\begin{array}{cc}
\int_{0}^{d_{1} \cdot T} i_{1}(t) \cdot d t & \int_{d_{1} \cdot T}^{T} i_{1}(t) \cdot d t \\
\vdots & \vdots \\
\int_{0}^{d_{N} \cdot T} i_{N}(t) \cdot d t & \int_{d_{N} \cdot T}^{T} i_{N}(t) \cdot d t
\end{array}\right] \cdot\left[\begin{array}{l}
R_{A} \\
R_{B}
\end{array}\right]} \\
& =\left[\begin{array}{c}
\int_{0}^{T} U_{1}(t) \cdot d t \\
\vdots \\
\int_{0}^{T} U_{N}(t) \cdot d t
\end{array}\right] \rightarrow\left[\begin{array}{l}
R_{A}^{*} \\
R_{B}^{*}
\end{array}\right]=\underline{\underline{D}}^{-1} \cdot \underline{u}
\end{aligned}
$$

In practical PWM applications, it is more convenient to change the duty ratio than the supply voltages because the supplies are usually constant, e.g. a battery. Having the elements of the coefficient matrix in (6) computed from average values (4), the noise suppression of the estimation is greatly improved. Drawback of the presented method is that the steady-state waveform is required which may take a long time to reach, especially if the control signals are changing. Furthermore, the duty ratio has to be always slightly altered in order to construct (6).

Considering embedded applications, the computational complexity of the presented method is low. In order to construct (6), the current waveform has to be integrated during the "on" and "off" periods of the PWM cycle respectively, and the average exciting voltage has to be calculated for a PWM cycle. The integration can be replaced by a finite summation, which is computed rapidly even in a low-end microcontroller, although the sampling frequency (number of samples in a PWM cycle) has to be high enough to reduce truncation error at the integration. The only computational difficulty may arise at solving the system of linear equations for the overall resistances (inversion).

\section{The Transient State Based Methods}

Compared to the previous section, the steady-state of the PWM is now not reached but the system undergoes a transient period due to a change in the control signal (duty ratio). However, in certain situations it might be still necessary to provide an estimate of the resistance, e.g. the transient period is long enough for the resistance to change considerably. In the following subsections 4.1 to 4.3 , a set of low-complexity computational methods are presented for the "transient" estimation of the coil's resistance. Unlike the method in section 3, the following methods can not directly scope with the nonlinear inductance behavior, which may pose some limitation with respect to practical applicability. However, this limitation can be mitigated as the inductance can be considered "almost" constant if the underlying range of the coil current, from which the estimation is performed, is not very large. In subsection 4.4, some further improvement is presented for reducing the bias if the inductance depends on the current.

With the modeling assumptions described in section 2, the estimation of the resistance is based on the following principle: if the supply voltages are constant and the duty ratio does not change, then the evolution of the "average current in a PWM cycle" follows the three parameter exponential function in (7) throughout the PWM cycles, even if the overall resistances in the energizing paths (time constants) are different. A mathematical proof of this statement is provided in the Appendix. Thus, full information about the underlying process (time constant and steady-state current) can be extracted from the average current waveform by e.g. fitting a three parameter exponential curve to the samples. In this paper, the resistance data are extracted by means of exponential fitting to the samples of the average current on a PWM cycle.

In the following methods, the basis of the estimation is the average current on a PWM cycle and not the current signal under a PWM cycle. The main reasons are noise suppression and computational effectiveness. By taking the average (integral) of the current on a PWM cycle, the noise which disturbs the measurement can be considerably reduced. The magnitude of the noise suppression is related to the number of samples in a PWM cycle (the higher the better), which can be considerably higher than the PWM switching frequency (e.g. 100 times), even in low-end microcontroller applications. The operation of averaging or integral, which is a summation, can be rapidly computed and require insignificant resources. Furthermore, the matrix operations, recursive and fitting methods, from which the resistance data are extracted e.g. exponential fitting in subsection 4.1-4.2, are thus to be performed on far less samples by considering the average current. Since these operations can be highly resource consuming (iteration, matrix inversion etc.) and their resource needs may nonlinearly increase with the sample size, their application to the average current signal (less samples) results in a significantly improved computational speed at the same or better signal to noise ratio. 
From the viewpoint of practical application, the upcoming methods require that: the sampling frequency has to be high enough (e.g. 100 times the frequency of the PWM) to satisfactorily compute the average current and average supply voltage on the PWM cycles, i.e., the discrete integration (summation) results in insignificant truncation error. Next, the duty ratio has to be held steady for at least three subsequent PWM cycles and the corresponding current and voltage signals are to be captured, so the exponential fitting can be performed. However, the more cycles (e.g. 8) available the more robust the estimation of the resistance becomes (better noise suppression). Additionally, at least two transient waveforms are necessary, which belong to different duty ratios so a system of linear equations, that is similar to (6), can be created. Using the concept of the exponential fitting, the method in subsection 4.1 extracts the resistances from the exponent, and the method in subsection 4.2 uses an extrapolation to the steady state of the current. The methods in subsection 4.3 bypass the exponential fitting and may also apply if the supply voltages change from PWM cycle to PWM cycle.

Further on, the following simplifying notations (8-11) are introduced; which are also better explained in the Appendix. The average current function, which can be taken only at the end of the PWM cycles, is derived as (7); and the steady-state of the average current can be derived as (8).

$$
i_{A V G, n}=i_{A V G}(n \cdot T)=\left(i_{A V G, 0}-i_{A V G}\right) \cdot e^{n \cdot T(A \cdot d+B \cdot(1-d))}+i_{A V G}
$$

$i_{A V G}=i_{H} \cdot d+i_{L} \cdot(1-d)+\frac{i_{H}-i_{L}}{T} \cdot \frac{\left(1-e^{a}\right) \cdot\left(1-e^{b}\right)}{1-e^{a+b}} \cdot\left(\frac{1}{A}-\frac{1}{B}\right)$

$$
\begin{gathered}
i_{H}=\frac{U_{H}}{R_{H}+R_{S}}=\frac{U_{H}}{R_{A}} \\
A=-\frac{R_{L}+R_{S}}{L_{S}}=-\frac{U_{A}}{R_{L}+R_{S}}=\frac{U_{L}}{R_{B}} \\
a=A \cdot d \cdot T \quad b=-\frac{R_{L}+R_{S}}{L_{S}}=-\frac{R_{B}}{L_{S}} \\
\end{gathered}
$$

\subsection{Estimation of the Resistance from the Exponent}

Under the conditions which are described in the introductory part of section 4, the average current is captured and computed for a few, e.g. six, successive PWM cycles; thus, (7) applies for the sampled average current signal. On the switching frequency, there is no limitation. By performing a three parameter exponential fit to the average current data by means of e.g. least squares, the resulting time constant $\Omega$ of the fitted exponential function can be expressed as (12) from (7) and (11).

$$
\Omega=(A-B) \cdot d+B \quad \rightarrow \quad \Omega(d)=m \cdot d+c
$$

According to (12), the fitted time constant $\Omega$ is a linear function of the duty ratio; and the intercept and the slope express the time constants of the energizing paths. If multiple transient waveforms (at least two), which belong to different duty ratios, are captured, then the linear relationship between the duty ratios and the fitted time constant in (12) can be identified; thus, the time constants can be estimated as in (13).

$$
B^{*}=c \quad A^{*}=m+c \quad \rightarrow \quad \frac{A^{*}}{B^{*}}=\frac{R_{A}}{R_{B}}
$$

By substituting (9-11) to (8) one can rewrite (8) as (14). With the previously identified time constants and resistance ratio in (13), the resistances of the energizing paths can be computed from (14). Also, the resistances $R_{A}$ and $R_{B}$ of the energizing paths can be computed from multiple values of (14) and then averaged to reduce noise disturbances.

$$
i_{A V G}=\left(\frac{U_{H}}{R_{A}}-\frac{U_{L}}{R_{B}}\right) \cdot\left[d+\frac{1}{T} \cdot \frac{\left(1-e^{a}\right) \cdot\left(1-e^{b}\right)}{1-e^{a+b}} \cdot\left(\frac{1}{A}-\frac{1}{B}\right)\right]+\frac{U_{L}}{R_{B}}
$$

Considering embedded applications, this method is computationally more exhaustive compared to the method in section 3 , because it requires an exponential function to be fitted to the average current waveform. This step may become time consuming in a microprocessor; therefore, efficient methods for performing a three parameter exponential fit may be of further research. Furthermore, the computation of (14) requires the computation of "exponential" functions which may also turn out to be multiple cycle instructions for a microcontroller.

\subsection{Extrapolation to the Steady-State of the Current}

In this subsection, the main concept is that the exponential fit is used for extrapolating to the steady-state of the average coil current, from which the overall resistances of the energizing paths can be extracted by using a simplified linear relationship (similarly to (6)). Compared to the previous method (subsection 4.1), this method requires that the time period $T$ of the PWM is considerably smaller (at least $1 / 10$ times) than the time constant of the electrical system.

Having an exponential curve fitted to a few successive samples of the transient average current, which is expressed as (7), the steady-state of the average current $i_{A V G}$ is also estimated by means of an e.g. exponential fitting. The analytical solution gives (14), which has to be compared to the steady state of the average current which resulted from the fitting. Since the resistance values are also contained in the time constant of the exponential terms, a closed form expression for the resistances from (14) does not exist but requires iteration, which is computationally exhaustive in embedded applications. Nonetheless, 
the value of the inductance also has to be approximated for evaluating (14). In order to bypass the previous difficulties, an approximation of (14) is proposed for the computation of the resistances. By taking the Taylor series of the exponential function in (15), the exponential part of (14) can be rearranged and simplified as in (16).

$$
\begin{gathered}
e^{x}=1+x+\frac{x^{2}}{2}+\frac{x^{3}}{6}+\frac{x^{4}}{24}+\ldots=\sum_{n=0}^{\infty} \frac{x^{n}}{n !} \\
\frac{\left(1-e^{a}\right) \cdot\left(1-e^{b}\right)}{1-e^{a+b}}=-\frac{\left(\sum_{n=1}^{\infty} \frac{a^{n}}{n !}\right) \cdot\left(\sum_{n=1}^{\infty} \frac{b^{n}}{n !}\right)}{\sum_{n=1}^{\infty} \frac{(a+b)^{n}}{n !}} \approx-\frac{a \cdot b}{a+b}
\end{gathered}
$$

According to (16), the parameters $a$ and $b$ express a ratio of the PWM time period and of the time constants in (11). For simplifying (14) and (16), we propose the use of the first order approximation of the exponentials, which we state that results in a very small truncation error of (16) in a wide range of the $a$ and $b$ parameters. With the assumption of $a=b$, the truncation error in (16) was computed at various values of the $a$ parameter, and the results are plotted in Fig. 4. It can be concluded that the first order approximation of (16) results in negligible error at values of the $a$ parameter typical in real PWM applications. According to Fig. 4, the error is still less than $10 \%$ at $a=1$ (time period of the PWM equals the time constant of the system); however, practical PWM applications use switching frequencies which are ten $(a=0.1)$ or more times higher, in which situations the truncation error becomes insignificant $(0.1 \%)$.

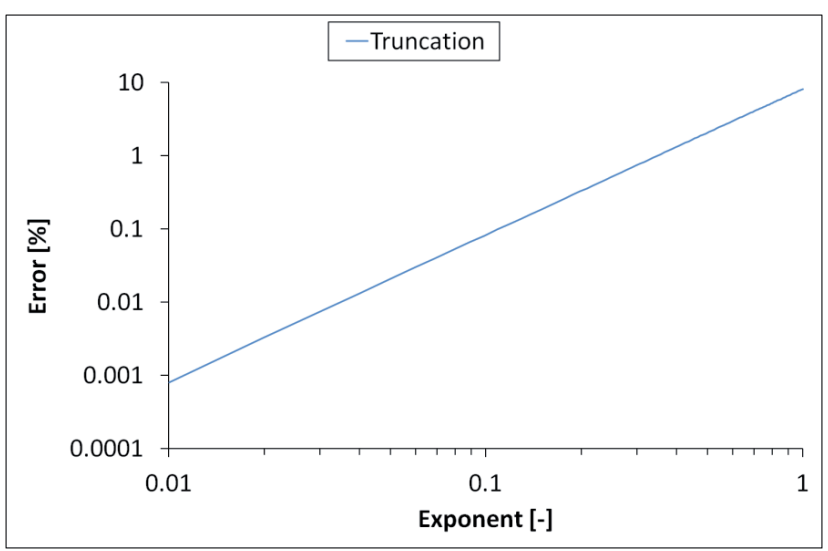

Fig. 4. Truncation error of (16) due to the first order approximation.

Using (16), the analytical solution of the average current (14) can be written as (17).

$$
i_{A V G}=\left(\frac{U_{H}}{R_{A}}-\frac{U_{L}}{R_{B}}\right) \cdot\left[d-\frac{1}{T} \cdot \frac{a \cdot b}{a+b} \cdot\left(\frac{1}{A}-\frac{1}{B}\right)\right]+\frac{U_{L}}{R_{B}}
$$

The term in the round brackets in (17) can be factored out as (18) using (10-11).

$$
\begin{aligned}
& d-\frac{1}{T} \cdot \frac{a \cdot b}{a+b} \cdot\left(\frac{1}{A}-\frac{1}{B}\right) \\
& =d+\frac{1}{T} \cdot \frac{\frac{R_{A}}{L_{S}} \cdot d \cdot T \cdot \frac{R_{B}}{L_{S}} \cdot(1-d) \cdot T}{\frac{R_{A}}{L_{S}} \cdot d \cdot T+\frac{R_{B}}{L_{S}} \cdot(1-d) \cdot T} \cdot\left(\frac{L_{S} \cdot R_{A}-L_{S} \cdot R_{B}}{R_{A} \cdot R_{B}}\right)
\end{aligned}
$$

From (18), the PWM time period $T$ and the inductance $L_{S}$ can be factored out thus the steady-state of the average current can be written as (19).

$$
i_{A V G}=\left(\frac{U_{H}}{R_{A}}-\frac{U_{L}}{R_{B}}\right) \cdot\left(d+\frac{d \cdot(1-d) \cdot\left(R_{A}-R_{B}\right)}{\left(R_{A}-R_{B}\right) \cdot d+R_{B}}\right)+\frac{U_{L}}{R_{B}}
$$

After a few algebraic manipulations (20-23) on (19), the resistances are brought to (24).

$$
\begin{gathered}
i_{A V G}=\left(\frac{U_{H}}{R_{A}}-\frac{U_{L}}{R_{B}}\right) \cdot d \cdot\left(1+\frac{(1-d) \cdot\left(R_{A}-R_{B}\right)}{\left(R_{A}-R_{B}\right) \cdot d+R_{B}}\right)+\frac{U_{L}}{R_{B}} \\
i_{A V G}=\left(\frac{U_{H}}{R_{A}}-\frac{U_{L}}{R_{B}}\right) \cdot d \cdot \frac{R_{B}}{\left(R_{A}-R_{B}\right) \cdot d+R_{B}}+\frac{U_{L}}{R_{B}} \\
i_{A V G}=\left(U_{H}-U_{L} \cdot \frac{R_{A}}{R_{B}}\right) \cdot \frac{d}{\left(R_{A}-R_{B}\right) \cdot d+R_{B}}+\frac{U_{L}}{R_{B}} \\
{\left[\left(R_{A}-R_{B}\right) \cdot d+R_{B}\right] \cdot i_{A V G}} \\
=U_{H} \cdot d+U_{L} \cdot\left(\frac{R_{A} \cdot d-R_{B} \cdot d+R_{B}}{R_{B}}-\frac{R_{A}}{R_{B}} \cdot d\right) \\
R_{A} \cdot d+R_{B} \cdot(1-d)=\frac{U_{H} \cdot d+U_{L} \cdot(1-d)}{i_{A V G}}
\end{gathered}
$$

If the extrapolations of the average current to steady-state are available at separate duty ratios, then a system of linear equations (25) can be established and solved for the overall resistances. The minimum number of independent equations is two; however, the system can be also composed of more equations for better noise suppression. Note that the problem in (25) is in fact a line fitting problem.

Compared to the method proposed in section 4.1, the method presented in section 4.2 is computationally more economical. In section 4.1, the solution requires an exponential fitting to the waveform of the average current, a line fitting to the time constant-duty ratio data and then the evaluation of (14) for every measurement point (to reduce noise), which can be time consuming for a microcontroller. However, the method 
in section 4.2 requires an extrapolation to the steady-state by the exponential fitting or other techniques, but then the resistances are directly obtained from the solution of (25), which is a line fitting. Thus, the computation of (14) is avoided and this increased computational efficiency can be of great advantage in embedded systems. Furthermore, the necessary matrix in (25) is constructed readily.

$$
\left[\begin{array}{ll}
d_{1} & 1-d_{1} \\
d_{2} & 1-d_{2}
\end{array}\right] \cdot\left[\begin{array}{l}
R_{A} \\
R_{B}
\end{array}\right]=\left[\begin{array}{l}
\frac{U_{H} \cdot d_{1}+U_{L} \cdot\left(1-d_{1}\right)}{i_{A V G, 1}} \\
\frac{U_{H} \cdot d_{2}+U_{L} \cdot\left(1-d_{2}\right)}{i_{A V G, 2}}
\end{array}\right]
$$

\subsection{Estimation from the Difference Equation}

The previous methods relied on an exponential fit to the average current signal (or on some other methods for obtaining the exponent and steady state); and required the supply voltages to remain constant during the period of an estimation. A recursive or fitting algorithm for obtaining the exponent and the steady state may pose a significant computational burden for a lowend microcontroller; therefore, this subsection presents alternative methods which bypass e.g. the exponential fitting; and may scope with changes in the supply voltages.

If considering the average current and voltage signals at a given duty ratio, the electrical behavior can be also represented by an equivalent resistance, e.g. the time constant in (7) and the "average" resistance in the right hand side of (25). The equivalent resistance, which is denoted by $R_{d}$, is a weighted combination of the $R_{A}$ and $R_{B}$ resistances by the $d$ duty ratio, provided the PWM frequency is high enough. According to subsection 4 , the samples of the average current can be considered as the samples of an otherwise continuous signal in (7), if the duty ratio and average supply voltage is unchanged. However, (7) is a closed form analytical solution to the corresponding differential equation, which can be described as in (26) in terms of the equivalent resistance $R_{d}$. In order to obtain (7) from (26), the average supply voltage has to be constant.

$$
L \cdot \frac{d i(t)}{d t}+R_{d} \cdot i(t)=U(t)
$$

Using (26), a system of linear equations (27) can be constructed from the measurement data and then solved for the unknown $L$ and $R_{d}$ values, provided that the duty ratio is constant. Note that by solving (27), it is possible to scope with changes in the average supply voltage; however, the derivative of the average current signal is necessary, which can be computed from the samples by using discrete differentiation as in (28). In the following, we denote the approach in (28) as "4.3 discr".

$$
\left[\begin{array}{cc}
\left.\frac{d i}{d t}\right|_{t 1} & i\left(t_{1}\right) \\
\vdots & \vdots \\
\left.\frac{d i}{d t}\right|_{t n} & i\left(t_{n}\right)
\end{array}\right] \cdot\left[\begin{array}{c}
L \\
R_{d}
\end{array}\right]=\left[\begin{array}{c}
U\left(t_{1}\right) \\
\vdots \\
U\left(t_{n}\right)
\end{array}\right]
$$

$$
\left[\begin{array}{cc}
\frac{i_{A V G, 1}-i_{A V G, 0}}{\Delta t} & \frac{i_{A V G, 1}+i_{A V G, 0}}{2} \\
\vdots & \vdots \\
\frac{i_{A V G, n}-i_{A V G, n-1}}{\Delta t} & \frac{i_{A V G, n}+i_{A V G, n-1}}{2}
\end{array}\right] \cdot\left[\begin{array}{c}
L \\
R_{d}
\end{array}\right]=\left[\begin{array}{c}
U_{A V G, 0} \\
\vdots \\
U_{A V G, n}
\end{array}\right]
$$

Despite being computationally economical, the solution of (28) is susceptible to noise and may also suffer from discretization error due to the discrete differentiation. If the average supply voltage does not change, then the average current signal is described by (7), for which an equivalent polynomial representation is possible. As an alternative approach, it is suggested that an e.g. $3^{\text {rd }}$ or $4^{\text {th }}$ order polynomial function (which reconstructs (7) appropriately) is fitted to the samples of the average current signal, and then the elements of (28) are computed from the polynomial representation. This way, the truncation error caused by the differentiation and sampling can be greatly reduced, and noise effects may be also decreased due to the polynomial fitting, although at the expense of the computational load of the polynomial fit and its evaluation. Further on, this approach is denoted as " 4.3 poly".

Nevertheless, it is also possible to recast (26) into an integral form which expresses the flux changes (29), thereby the discrete differentiation is bypassed. Note that this approach can also scope with changes in the supply voltage, but the computation of the coefficient matrix (29) becomes more resource consuming compared to (28). The parameters can be derived by solving the resulting system of linear equations (the elements of (27) to be replaced accordingly to (29)). This approach is denoted as "4.3 int".

$$
\begin{aligned}
& \int L \cdot \frac{d i(t)}{d t} \cdot d t+R_{d} \cdot \int i(t) \cdot d t=\int U(t) \cdot d t \\
& L \cdot \frac{i_{n}-i_{0}}{n \cdot T}+R_{d} \cdot\left(\sum_{k=0}^{n} i_{A V G, k}-\frac{i_{A V G, n}+i_{A V G, 0}}{2}\right) \\
& =\sum_{k=0}^{n} U_{A V G, k}-\frac{U_{A V G, n}+U_{A V G, 0}}{2}
\end{aligned}
$$

After having the equivalent resistance $R_{d}$ estimated at different duty ratios, the overall resistances of the energizing paths can be estimated accordingly to e.g. (25). Compared to the methods in subsections 4.1-4.2, the (exponential) fitting procedure is avoided, which may result in a considerable improvement at the computational efficiency. However, the solution requires the inversion of two matrices: one for the equivalent resistance (e.g. (28)) and the other for the overall resistances (25). 
The statistical properties, i.e., mean and variance of the estimates of the equivalent resistance from (14), (25), (27)-(29) are expected to somewhat depend on the coefficient matrix; therefore, an experimental investigation is conducted in Section 5.

\subsection{Reducing Bias in the Estimate of the Resistance}

In the introductory part of section 4 , it is pointed out that the nonlinear core behavior may cause bias in the estimation because the underlying model considers a linear, constant inductance. In this subsection, an approach is presented for the reduction of this bias.

The methods in subsections 4.1 to 4.2 are based on estimating the steady-state current from an exponential fit to the transient step response. In real applications, the inductance of the solenoid valve might somewhat depend on the current; therefore, the exponential extrapolation that considers a constant inductance becomes biased. The exponential function in (7), which is expressed in terms of amplitude and offset, can be rewritten as (30) in terms of initial current and steady-state.

$$
i(t)=i_{0}+\left(i_{S S}-i_{0}\right) \cdot(1-\exp (A \cdot t))
$$

When performing an exponential fit to the measurement data, it can be also considered as finding an appropriate initial current $i_{0}$ and current change $i_{S S}-i_{0}$. The term $i_{S S}$ refers to the steady-state current. Because the initial current is known (measured), it is expected to change far less compared to $i_{S S}-i_{0}$ and $A$ in case of bias due to a current dependent inductance. Assume that the inductance function is such that the $i_{S S}-i_{0}$ term in (30) is over estimated. In this situation, if the current (duty ratio) increases, then the aforementioned bias causes that the extrapolated steady-state current becomes larger than the original one; which corresponds to an estimated resistance being smaller than the real one. On the contrary, if the current (duty ratio) decreases, then the larger (biased) $i_{S S}-i_{0}$ term causes the extrapolation to the steady current to become smaller; thus, the corresponding resistance will be overestimated. This is opposite to the previous situation. That is how, if adding the biased resistances (previous two situations) to each other, the over and under estimations will somewhat cancel out each other and will thus result in a considerably smaller overall bias.

For reducing the overall bias in the steady-state (resistance) estimate due to a current dependent inductance, we propose that (25) be constructed from "averaged" terms as in (31). Considering (24), the right-hand side represents an average or equivalent resistance, which is either over or under estimated in case the inductance is not constant. Mathematically, the separate (independent) rows of (25) can be added to each other and averaged as in (31).

$$
R_{A} \cdot \frac{1}{N} \cdot \sum_{n=1}^{N} d_{n}+R_{B} \cdot \frac{1}{N} \cdot \sum_{n=1}^{N}\left(1-d_{n}\right)=\frac{1}{N} \cdot \sum_{n=1}^{N} R_{n}
$$

If the equivalent resistances, which are computed from falling and rising current signals at different duty ratios, are added to each and averaged as in (31), then the overall bias in the "averaged" equivalent resistance can be considerably reduced. Eq. (31) can be rewritten as (32).

$$
\left(R_{A}-R_{B}\right) \cdot \overline{d_{N}}+R_{B}=\overline{R_{N}}
$$

According to (32), the average resistance is described as a linear function of the average duty ratio, where the slope and the intercept are simple functions of the resistances in the energizing paths. Therefore, a matrix equation similar to (24) can be constructed and solved, or the linear relationship identified from multiple average duty ratio and average resistance pairs.

\section{Computer Simulations and Experimental Analyses 5.1 Computer simulations for comparing some statistical properties:}

In Section 4 a collection of methods was presented for estimating the overall resistances in the energizing paths considering the transient current waveform. Common in them all, an equivalent resistance is estimated at a certain duty ratio; then a system of linear equations is created from the equivalent resistances and solved for the resistances in the energizing paths. In a computer simulation, we studied the mean error and deviation of each of the methods in section 4 when estimating a certain equivalent resistance. The parameters of the simulation were as follows: $L=1, R_{A}=0.7, R_{B}=0.55, U_{H}=1, U_{L}=0, T=0.2, T_{s}=0.002$, $d=0.4, i_{0}=0$ and 10 PWM cycles were recorded. The average current signal was ranging from 0 to 0.45 . The samples of the current signal were loaded with a uniformly distributed white noise with expected value of 0 . The simulation was carried out at different noise levels. At each of the noise amplitudes, 2048 independent simulations were preformed from which an experimental mean error and deviation were computed for the separate methods. The performance of the proposed methods versus the noise disturbances is plotted in Figs. 5-6. The equivalent resistance in this particular simulation was 0.61 .

From left to right, the labels in the legends are the following: "4.1" refers to the method that is proposed in section 4.1 (exponential fit to get the time constant), "4.2" refers to the method that is proposed in section 4.2 (exponential fit to get the steady-state), "4.3.discr" refers to the method that is proposed in section 4.3 and uses the discrete form of (27) that is (28); "4.3.int" refers to the method which uses the integral form of (27) that is (29) and "4.3.poly" refers to the method which uses a polynomial $\left(4^{\text {th }}\right.$ order) fit to the average current waveform and thus computes (27). According to Fig. 5, the "discrete" version of the method that is proposed in section 4.3 (from (28)) has a significant bias with increasing noise levels. Compared to this, the integral form (29) in Section 4.3 is "unbiased"; however, it has the largest deviation. Therefore, if a method that is computationally more effective than those in Section 4.1-4.2 are necessary, we suggest that (27) 

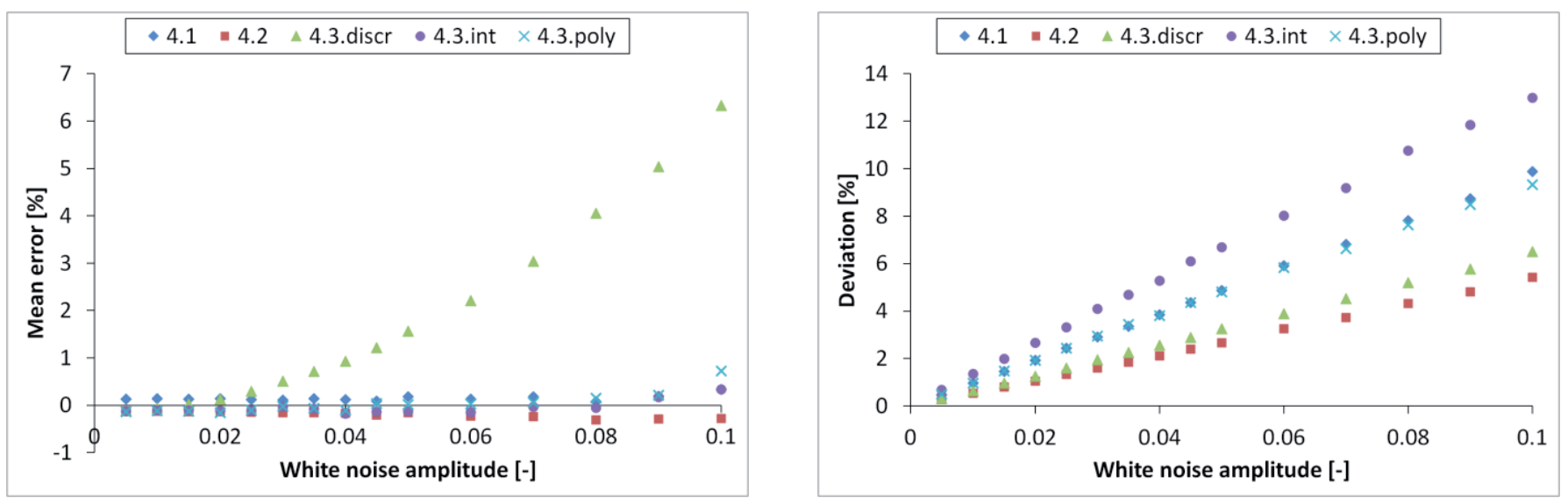

Fig. 5. Mean error and deviation of the proposed methods

Tab. 1. Results of resistance estimations.

\begin{tabular}{|c|c|c|c|c|c|c|}
\hline & $\begin{array}{c}\text { DC } \\
\text { Meas. }\end{array}$ & $\begin{array}{c}\text { Sec. } 3 \\
\text { St. state (4) }\end{array}$ & $\begin{array}{c}\text { Sec. } 4.2 \\
\text { Exp.fit (32) }\end{array}$ & $\begin{array}{c}\text { Sec. } 4.3 \\
\text { discr. (28) }\end{array}$ & $\begin{array}{l}\text { Sec. } 4.3 \\
\text { int. (29) }\end{array}$ & $\begin{array}{l}\text { Sec. } 4.3 \\
\text { poly. (27) }\end{array}$ \\
\hline $\mathbf{R}_{\mathrm{A}}[\Omega]$ & 6.117 & 6.137 & 6.119 & 6.130 & 6.110 & 6.107 \\
\hline $\mathbf{R}_{\mathrm{B}}[\Omega]$ & 5.755 & 5.765 & 5.736 & 5.734 & 5.740 & 5.754 \\
\hline$\Delta R[\Omega]$ & 0.362 & 0.372 & 0.383 & 0.396 & 0.371 & 0.353 \\
\hline
\end{tabular}

is implemented by computing the coefficients from an adequate polynomial fit to the average current signal, i.e., use "4.3.poly".

Nonetheless, the best statistical performance (bias and deviance) is shown by the method in subsection 4.2; however, it is computationally more demanding as it requires an exponential fitting compared to the methods in 4.3 .

\subsection{Experimental analyses}

The resistance of a real solenoid actuator was estimated with the methods that are proposed in Sections 3-4. The experimental setup that was used for the analyses is described in [13]. The measurement settings were the following: $100 \mathrm{kHz}$ sampling frequency, $2 \mathrm{kHz}$ switching frequency, $10 \mathrm{~V} U_{H}$ high level power supply and the $U_{L}$ low level or negative supply corresponded to the forward voltage drop of the freewheeling diode, the model of which had been previously captured. The solenoid actuator was driven in a low-side single switch configuration similarly to Fig. 1. The overall resistances had been previously measured in a DC static measurement (the switch was continuously turned on); the results are listed in Table 1.

First, the overall resistances in the energizing paths (which include the coil's resistance) were estimated from the steadystate PWM current waveform accordingly to Section 3. The duty ratio was swept from 0.3 to 0.4 with a spacing of 0.02 and the current waveform during a PWM cycle was recorded after $100 \mathrm{~ms}$ the duty ratio had changed, thus steady-state was reached. The resulting system of linear equations (6) was solved that yielded the resistances in Table 1.

Secondly, the overall resistances were estimated from the transient average current signal accordingly to subsections 4.2-4.3.
Two sequences of duty ratios, each consisting of six different duty ratios, were used for generating the exciting PWM signal and a duty ratio lasted for approximately 18 PWM cycles. The corresponding transient waveforms are plotted in Fig. 6. For the separate transient sections that corresponded to a certain duty ratio, the steady-state current was approximated by means of an exponential fit to the last six measurement points or using (27)-(29); and then the average duty ratios and resistances (32) were calculated for the two sequences. Thus, bias due to a possibly current dependent inductance could be considerably reduced. The resistance estimates were obtained by solving the 2 by 2 system of equations (25).

According to Table 1, the difference in the overall resistances could be estimated appropriately with the proposed methods. Thus, a better estimate of the resistance of the solenoid actuator could be provided compared to computing a single "equivalent" resistance, which would also change with the duty ratio; thus, result in a bias that depends on the duty ratio.

\section{Conclusion}

In this paper, methods were developed for estimating the resistance of the coil of a solenoid actuator by considering the fact that in real applications, the overall resistance that the coil's current encounters during the "on" and "off" periods of a PWM cycle can be different. In some situations, the difference in the overall resistances can be significant; thus, we provided better estimates of the coil's resistance and resolved a possible source of bias, compared to estimating a single equivalent resistance. Also, we differentiated between estimating the resistance from the steady and transient waveforms of the PWM driven current 

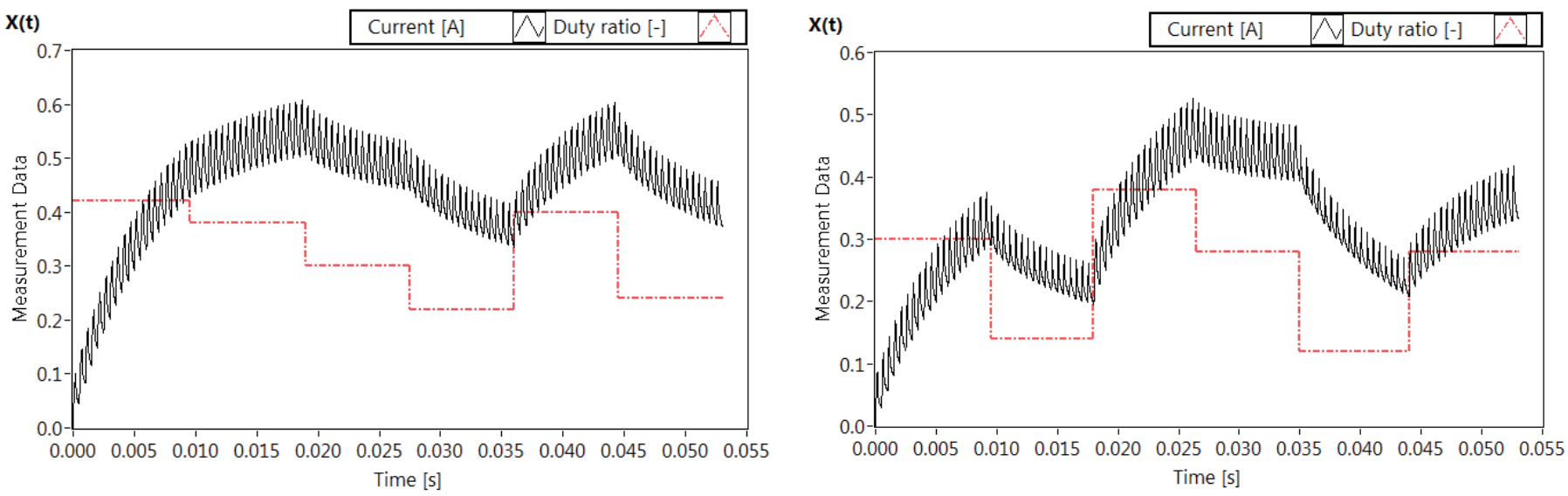

Fig. 6. Transient current waveforms of the solenoid actuator used for resistance estimation.

signal. In the latter case, we proved that under some modeling assumptions the transient waveform of the average current on a PWM cycle could be expressed by a three parameter exponential function irrespectively of the different overall resistances (time constants). Computer simulations had been carried out to compare the statistical properties, e.g. expected error and deviance, of the developed methods. We found that in case the exponential assumption is valid, the estimation of the resistance by the exponential fitting methods had better statistical properties. However, computation of the exponential fitting may become computationally exhaustive for certain microcontroller based applications; therefore, the methods in subsection 4.3, which are computationally more economical, can be more suitable provided that noise effects are at an acceptable level. Experimental analyses that considered a real actuator also confirmed that the proposed methods (steady and transient) could estimate the resistance of the solenoid actuator appropriately; and that some difference in the overall resistances of the energizing paths was observable. Thus, a better estimate of the true winding resistance could be provided and bias in the estimate of the resistance, which would otherwise change with the duty ratio, could be reduced. In general, the developed methods have low computational complexity which makes them suitable for embedded applications.

\section{Appendix}

We prove the principle in section 4: if the inductance is constant, then the step response of the average current on a PWM cycle can be expressed as (7) if the time constants in the PWM "on" and "off" periods are different. During a PWM "on" period (switch is turned on) and during a PWM "off" period (switch is turned off) the coil current can be expressed as (a.1)(a.2) respectively according to Fig. 1-2. The terms $i_{A, 0}$ and $i_{B, 0}$ in (a.1)-(a.2) refer to the initial conditions of the coil current. The following notations (9)-(11) are also made use of.

$$
i_{O N}(t)=\left(i_{A, 0}-i_{H}\right) \cdot e^{A \cdot t}+i_{H}
$$

$$
i_{\text {OFF }}(t)=\left(i_{B, 0}-i_{L}\right) \cdot e^{B \cdot t}+i_{L}
$$

For a particular $k^{\text {th }}$ PWM cycle the following notations (a.3)(a.5) are introduced for the current signal. At the beginning of the PWM cycle ("on" period) the initial or start current is denoted as $i_{A, k}$. Next, the symbol $i_{B, k}$ refers to the current that is reached by the end of the $k^{\text {th }}$ PWM "on" period. The coil current at the end of the $k^{\text {th }}$ PWM cycle, which is the start current of the next $k+1^{\text {th }}$ PWM cycle, is denoted by $i_{A, k+1}$. Using (a.1)(a.2) the specific current values in an arbitrary PWM cycle can be expressed as (a.3)-(a.5).

$$
\begin{gathered}
i_{B, k}=\left(i_{A, k}-i_{H}\right) \cdot e^{A \cdot d \cdot T}+i_{H} \\
i_{A, k+1}=\left(i_{B, k}-i_{L}\right) \cdot e^{B \cdot(1-d) \cdot T}+i_{L} \\
i_{A, k+1}=i_{A, k} \cdot e^{a+b}-i_{H} \cdot e^{a+b}+\left(i_{H}-i_{L}\right) \cdot e^{b}+i_{L} \\
\alpha=-i_{H} \cdot e^{a+b}+\left(i_{H}-i_{L}\right) \cdot e^{b}+i_{L}
\end{gathered}
$$

Using the formulas (a.5)-(a.6) successively, the initial current of the PWM cycles can be expressed as (a.7) from an arbitrary start current $i_{A, k}$.

$$
\begin{aligned}
& i_{A, k+1}=i_{A, k} \cdot e^{a+b}+\alpha \\
& i_{A, k+2}=i_{A, k} \cdot e^{2 \cdot(a+b)}+e^{a+b} \cdot \alpha+\alpha \\
& \ldots \ldots \ldots \\
& i_{A, k+n}=i_{A, k} \cdot e^{n \cdot(a+b)}+\alpha \cdot \sum_{j=0}^{n-1} e^{j \cdot(a+b)}
\end{aligned}
$$

According to (a.7), the evolution of the current, which is taken at the start of the PWM cycles, follows a geometric series. Using the formulae for the sum of geometric series, the summation can be transformed to (a.8).

$$
i_{A, k+n}=i_{A, k} \cdot e^{n \cdot(a+b)}+\alpha \cdot \frac{1-e^{n \cdot(a+b)}}{1-e^{a+b}}
$$

Through the substitution and rearrangement of the coefficients, (a.8) can be brought to (a.9) which possesses the form 
of (7); that is, the initial current of the PWM cycles can be expressed by the exponential relationship irrespective of the different time constants. Though the initial current is sampled by the PWM switching frequency, it still fits an otherwise continuous exponential signal. The overall time constant is a weighted sum of the time constants.

$$
i_{A, k+n}=\left(i_{A, k}-\frac{\alpha}{1-e^{a+b}}\right) \cdot e^{n \cdot(a+b)}+\frac{\alpha}{1-e^{a+b}}
$$

In Section 4, the resistances are estimated from the average current in a PWM cycle. In terms of $i_{A, k}, i_{B, k}$ and $i_{A, k+1}$ the average current in the $k^{\text {th }}$ PWM cycle can be written as (a.10)-(a.11).

$i_{A V G, k}=\frac{\int_{0}^{d \cdot T}\left[\left(i_{A, k}-i_{H}\right) \cdot e^{A \cdot t}+i_{H}\right] \cdot d t+\int_{0}^{(1-d) \cdot T}\left[\left(i_{B, k}-i_{L}\right) \cdot e^{B \cdot t}+i_{L}\right] \cdot d t}{T}$

$$
\begin{aligned}
i_{A V G, k}= & \frac{i_{A, k}-i_{H}}{A \cdot T} \cdot\left(e^{a}-1\right)+i_{H} \cdot d \\
& +\frac{i_{B, k}-i_{L}}{B \cdot T} \cdot\left(e^{b}-1\right)+i_{L} \cdot(1-d)
\end{aligned}
$$

By substituting (a.2) to (a.11), the average current can be rearranged as in (a.12).

$$
\begin{aligned}
i_{A V G, k} & =\frac{i_{A, k}-i_{H}}{A \cdot T} \cdot\left(e^{a}-1\right) \\
& +\frac{\left(i_{A, k}-i_{H}\right) \cdot e^{a}+i_{H}-i_{L}}{B \cdot T} \cdot\left(e^{b}-1\right) \\
& +i_{H} \cdot d+i_{L} \cdot(1-d) \\
& =i_{H} \cdot\left[d+\left(1-e^{a}\right) \cdot\left(\frac{1}{A \cdot T}-\frac{1-e^{b}}{B \cdot T}\right)\right] \\
& +i_{L} \cdot\left[1-d+\frac{1-e^{b}}{B \cdot T}\right] \\
& +i_{A, k} \cdot\left(\frac{e^{a}-1}{A \cdot T}+\frac{e^{b}-1}{B \cdot T} \cdot e^{a}\right)
\end{aligned}
$$

\section{References}

1 Rashed M., Sensorless Indirect-Rotor-Field-Orientation Speed Control of a Permanent-Magnet Synchronous Motor with Stator-Resistance Estimation. IEEE Transactions on Industrial Electronics, 54 (3), pp. 1664-1675, (2007).

DOI: $\underline{\text { 10.1109/TIE.2007.895136 }}$

2 Finch J. W., Giaoruris D., Controlled AC Electrical Drives. IEEE Transactions on Industrial Electronics, 55 (2), pp. 481-491, (2008).

3 Wilson S. D., Stewart P., Taylor B. P., Methods of Resistance Estimation in Permanent Magnet Synchronous Motors for Real-Time Thermal Management. IEEE Transactions on Energy Conversion, 25 (3), pp. 698-707, (2010). DOI: $10.1109 /$ TEC.2010.2051811

Introducing the following notations (a.13)-(a.14), (a.12) can be rewritten as (a.15).

$$
\begin{gathered}
\beta=i_{H} \cdot\left[d+\left(1-e^{a}\right) \cdot\left(\frac{1}{A \cdot T}-\frac{1-e^{b}}{B \cdot T}\right)\right]+i_{L} \cdot\left[1-d+\frac{1-e^{b}}{B \cdot T}\right] \\
\chi=\frac{e^{a}-1}{A \cdot T}+\frac{e^{b}-1}{B \cdot T} \cdot e^{a} \\
i_{A V G, k}=\chi \cdot i_{A, k}+\beta
\end{gathered}
$$

According to (a.15), the average current in the $k^{\text {th }}$ PWM cycle is a linear combination of its corresponding start current. Using the previously established exponential expression (a.9) for the evolution of the start current values, the average current can be expressed as (a.16).

$$
i_{A V G, k+n}=\chi \cdot\left(i_{A, k}-\frac{\alpha}{1-e^{a+b}}\right) \cdot e^{n \cdot(a+b)}+\frac{\chi \cdot \alpha}{1-e^{a+b}}+\beta
$$

From (a.16), it can be seen that the evolution of the average coil current, similarly to the start current in (a.9), can be also represented as the samples of a continuous exponential function. Substituting (a.6), (a.13) and (a.14); the steady state of the average current can be expressed as (8) with $n$ set to infinity, provided that $a$ and $b$ are negative.
4 Rahman M. F., Cheung N. C., Lim K. W., Position estimation in solenoid actuators. IEEE Industry Applications, 32 (3), pp. 552-559, (1996). DOI: 10.1109/IAS.1995.530338

5 Renn J-C., Chou Y-S., Sensorless Plunger Position Control for a Switching Solenoid. JSME International Journal Series C, 47 (2), pp. 637-645, (2004).

DOI: $10.1299 / \mathrm{jsmec} .47 .637$

6 Wu S-T., Chen W-N., Self-sensing of a solenoid valve via phase detection. In Proceedings IEEE/ASME International Conference on Advanced Intelligent Mechatronics, pp. 1165-1170, (2009). DOI: $10.1109 /$ AIM.2009.5229744 
7 Maridor J., Katic N., Perriard Y., Ladas D., Sensorless position detection of a linear actuator using the resonance frequency. In ICEMS Electrical Machines and Systems. pp. 1-6, (2009).

DOI: $\underline{10.1109 / I C E M S .2009 .5382641}$

8 Chladny R. R., Koch C. R., Flatness-Based Tracking of an Electromechanical Variable Valve Timing Actuator With Disturbance Observer Feedforward Compensation. IEEE Transactions on Control Systems Technology, 16 (4), pp. 652-663, (2008).

DOI: $10.1109 /$ TCST.2007.912121

9 Espinosa A. G., Riba Ruiz J.-R. , Cusidó J., Morera X. A., Sensorless Control and Fault Diagnosis of Electromechanical Contactors. IEEE Transactions on Industrial Electronics, 55 (10), pp. 3742-3750, (2008).

DOI: 10.1109/TIE.2008.925773
10 Cai B., Liu Y., Tian X., Wang Z., Wang F., Li H., Ji R., Optimization of submersible solenoid valves for subsea blowout preventers. IEEE Transactions on Magnetics, 47 (2), pp. 451-458, (2011). DOI: 10.1109/TMAG.2010.2100825

11 Eyabi P., Washington G., Modeling and sensorless control of an electromagnetic valve actuator. Mechatronics, 16 (3-4), pp. 159-175, (2006).

DOI: $10.1016 /$ j.mechatronics.2005.11.008

12 Jung H. G., Hwang J. Y., Yoon P. J., Kim J. H., Resistance estimation of a PWM-driven solenoid. International Journal of Automotive Technology, 8 (2), pp. 249-258, (2007).

13 Dülk I., Kovácsházy T., A novel experimental setup for solenoid actuators. In Proceedings IEEE IECON. pp. 3862-3867, (2013). DOI: 10.1109/IECON.2013.6699752 